\title{
Role of Cholesterol on Binding of Amyloid Fibrils to Lipid Bilayers.
}

Cristiano L. Dias ${ }^{1}$, Sharareh Jalali ${ }^{1}$, Yanxing Yang ${ }^{1}$, and Luis Cruz $^{2}$

${ }^{1}$ Physics Department, New Jersey Institute of Technology, Newark, New Jersey, 07102-1982 USA

${ }^{2}$ Department of Physics, 3141 Chestnut Street, Drexel University, Philadelphia, Pennsylvania 19104 USA 


\section{S1) Description of the different simulation setups}

Thirty (30) different simulations were performed in this study. Each simulations is $6 \mu$ s long which accounts for a total of $180 \mu$ s of simulation time. The list of all simulations is shown in Table S1.

\begin{tabular}{|c|c|c|c|c|c|c|c|c|}
\hline \multirow{2}{*}{ Fibril } & \multirow{2}{*}{$\begin{array}{l}\text { Lipid composition } \\
\text { of bilayer }\end{array}$} & \multicolumn{4}{|c|}{ Number of } & \multirow{2}{*}{$\begin{array}{c}\text { Num. } \\
\text { of } \\
\text { sim. }\end{array}$} & \multirow{2}{*}{$\begin{array}{l}\text { Length } \\
\text { of each } \\
\text { sim. }\end{array}$} & \multirow{2}{*}{$\begin{array}{l}\text { Average box size } \\
(\mathrm{nm} \times \mathrm{nm} \times \mathrm{nm})\end{array}$} \\
\hline & & DPPC & DTPT & CHOL & Water & & & \\
\hline \multirow{3}{*}{ 2BEG } & $100 \% \mathrm{PC}$ & 578 & - & - & 35,757 & 4 & $6 \mu \mathrm{s}$ & $13.8 \times 13.8 \times 27.6$ \\
\hline & $90 \% P C+10 \%$ PA & 520 & 56 & - & 35,090 & 4 & $6 \mu \mathrm{s}$ & $13.6 \times 13.6 \times 27.9$ \\
\hline & $70 \% \mathrm{PC}+30 \% \mathrm{CHOL}$ & 404 & - & 172 & 26,656 & 4 & $6 \mu \mathrm{s}$ & $12 \times 12 \times 27.5$ \\
\hline \multirow{4}{*}{$2 \mathrm{MXU}$} & $100 \% \mathrm{PC}$ & 578 & - & - & 35,574 & 4 & $6 \mu \mathrm{s}$ & $13.8 \times 13.8 \times 27.6$ \\
\hline & $90 \% P C+10 \%$ PA & 520 & 56 & - & 34,902 & 4 & $6 \mu \mathrm{s}$ & $13.6 \times 13.6 \times 27.8$ \\
\hline & $70 \% \mathrm{PC}+30 \% \mathrm{CHOL}$ & 404 & - & 172 & 26,429 & 4 & $6 \mu \mathrm{s}$ & $12 \times 12 \times 27.4$ \\
\hline & $50 \% \mathrm{PC}+50 \% \mathrm{CHOL}$ & 288 & - & 288 & 22,751 & 6 & $6 \mu \mathrm{s}$ & $11 \times 11 \times 28.2$ \\
\hline
\end{tabular}

Table S1 - Details of the different simulation setups 
S2) Minimal distance between fibril and $\mathrm{PO}_{4}$ beads for the different systems studied here

Figures in this section were computed as described in the main text for Figure 1d,e. Data from these figures were used to compute the table in Fig. $1 \mathrm{~h}$.

\section{S2.1) PC bilayers}

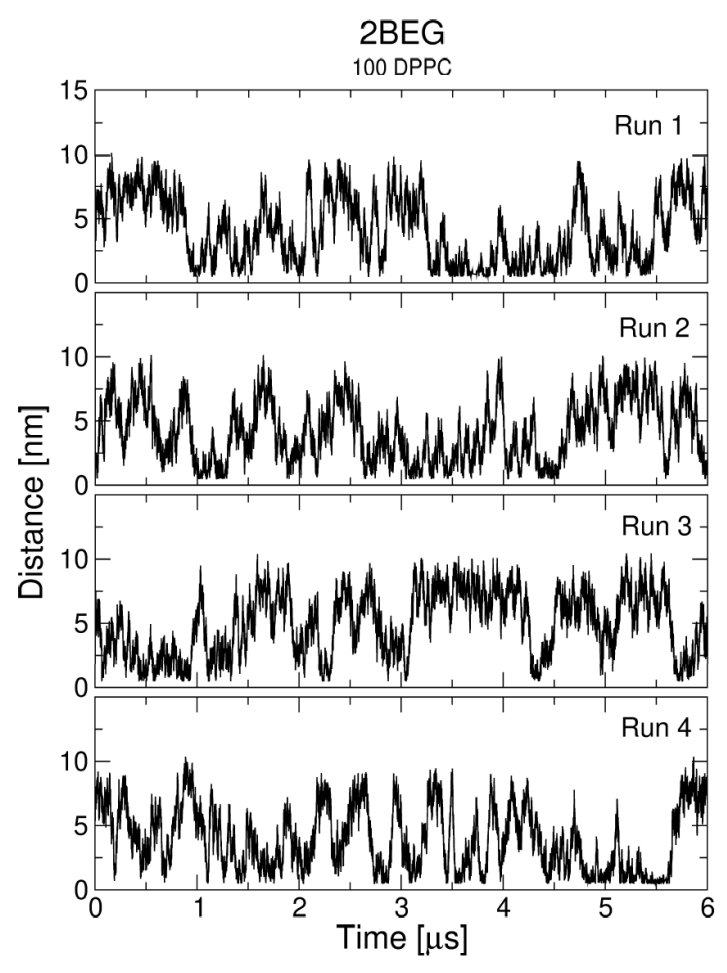

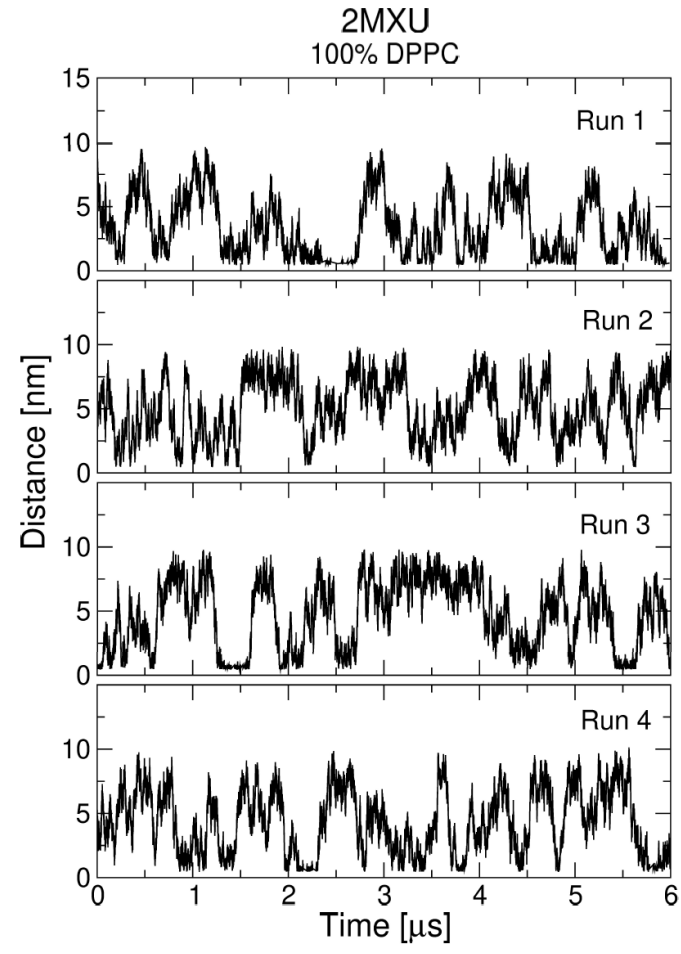




\section{S2.2) $90 \%$ PC + 10\% PA}

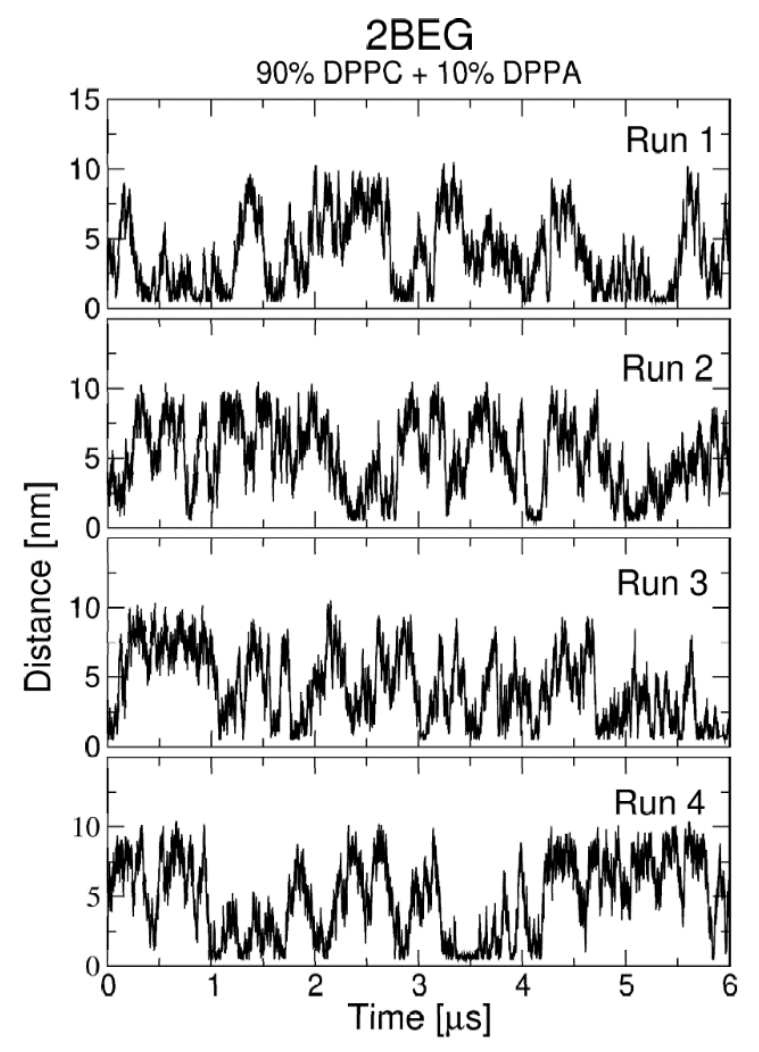

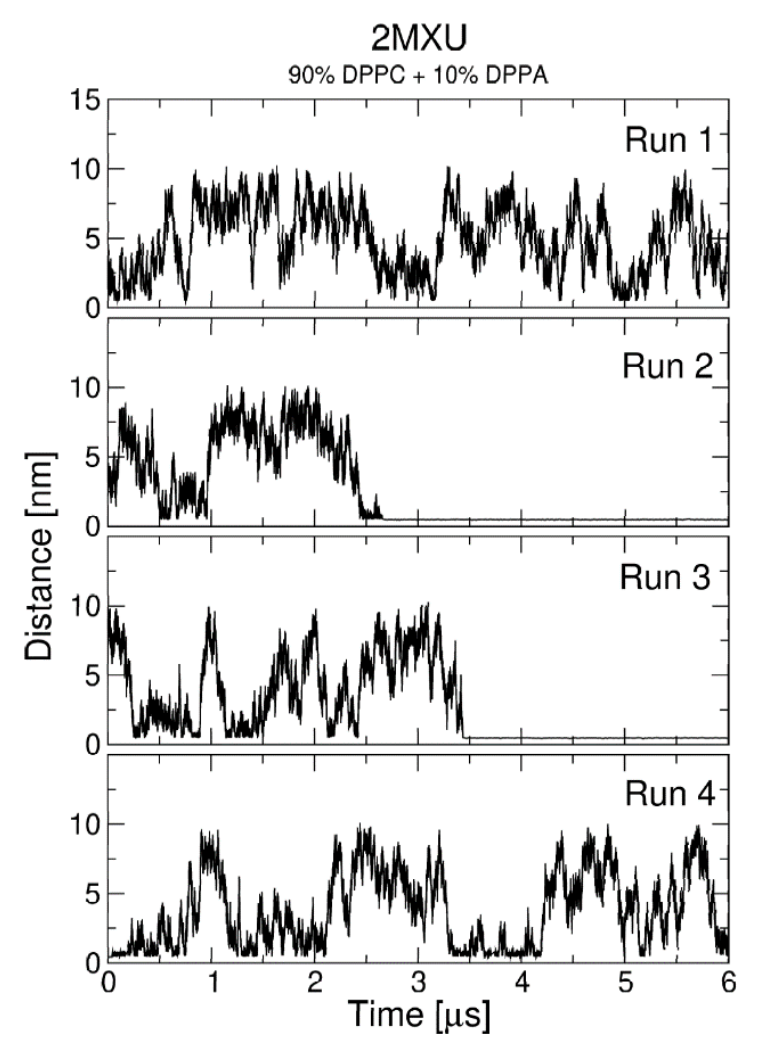



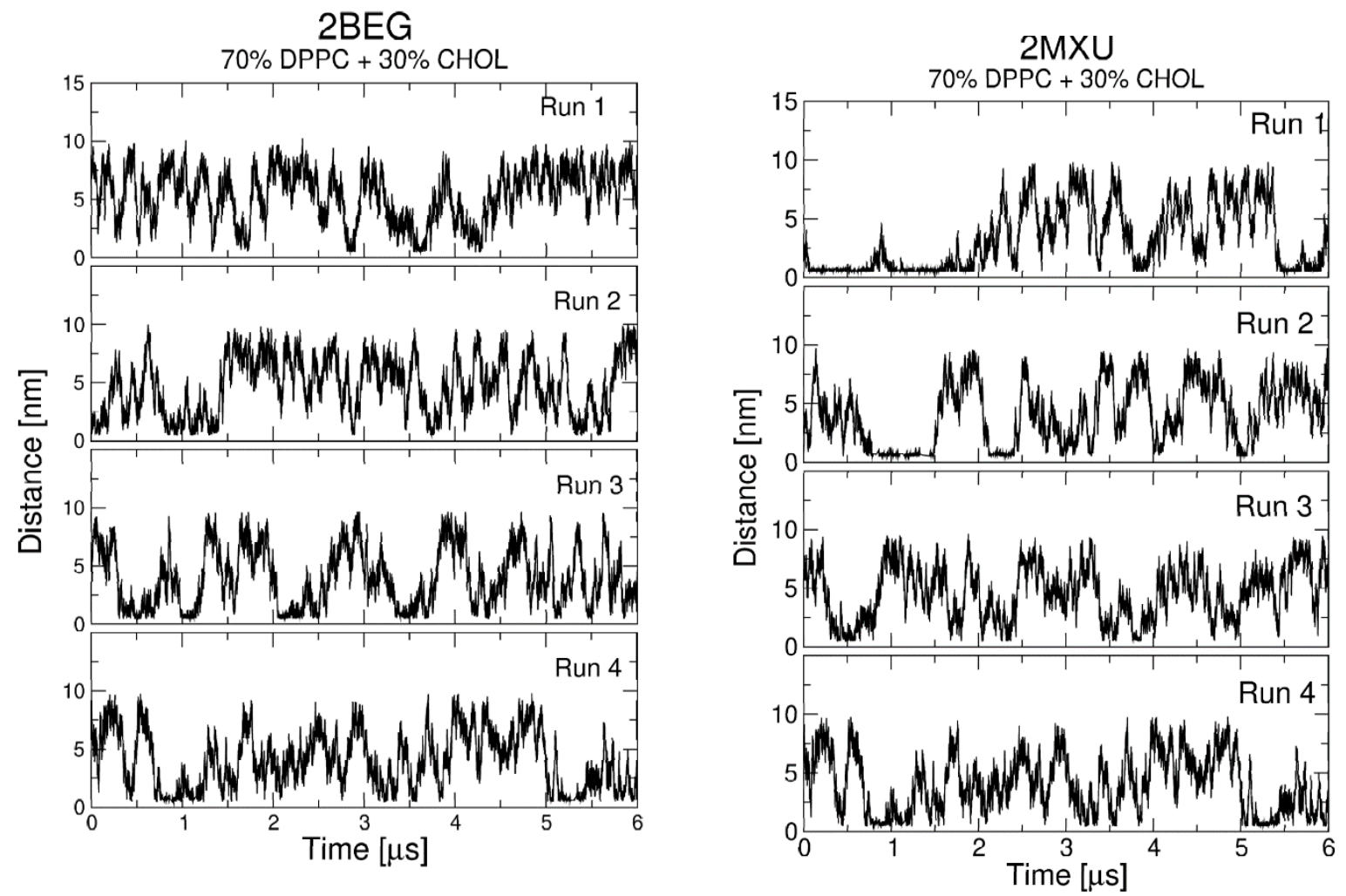

\section{S2.4) $50 \% \mathrm{PC}+50 \% \mathrm{CHOL}$}

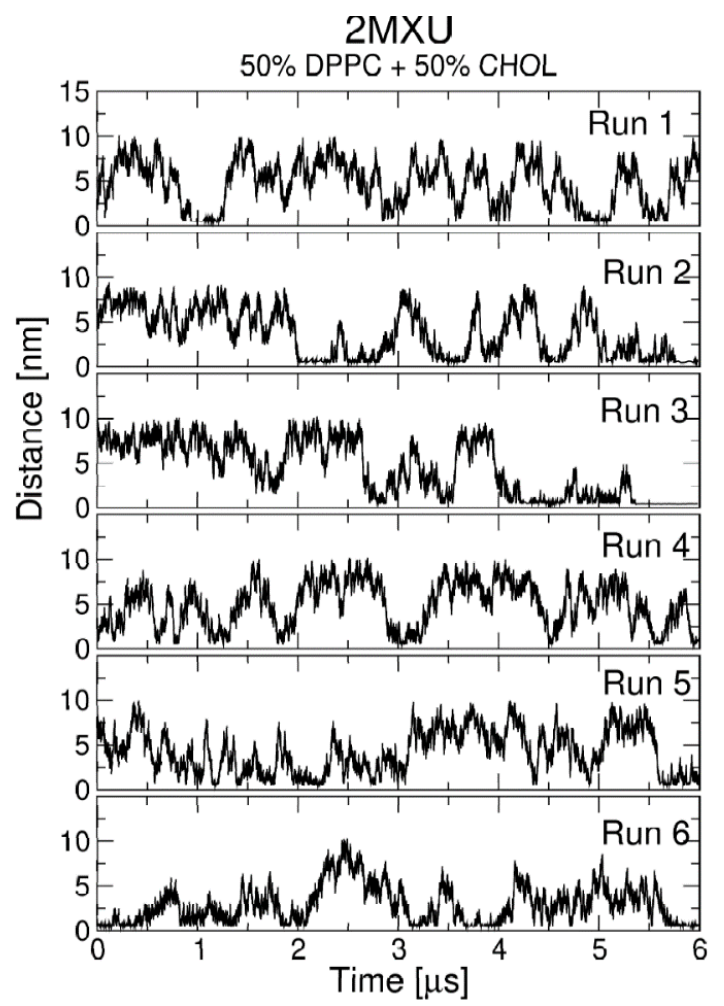




\section{S3) Effects of cholesterol on the DPPC lipids}

In the main text, we show that electrostatic interactions play an important role in accounting for binding of amyloid fibrils to lipid bilayers. Thus, changes in the density and/or orientation of charged lipid beads with cholesterol concentration can play an importat role in this process. This is studied in Fig. S3.

\section{S3.1) Area per PC lipid as a function of cholesterol concentration}

The addition of cholesterol to bilayers affects the surface density of lipid charged beads. This is usually reported in the literature as the total area of the bilayer divided by the number of PC lipids, i.e., Area/NPC. All-atom simulations of POPC bilayers performed using different force fields (see Fig. S14 of reference [4]) have reported that Area/NPC increases monotonically with increasing cholesterol concentration. A similar results was also obtained for DMPC [3]. For most lipids, Area/NPC is larger at $50 \%$ cholesterol content than at $0 \%$ (see for example table 3 of reference [3]). However, cholesterol was also reported to account for a reduction in Area/NPC at small cholesterol concentrations for simulations of DPPC [1] and DOPC [2]. These results are reproduced in Fig. S14. In our simulations (in red), Area/NPc increases monotonically implying that the density of head groups decreases with increasing cholesterol concentration. This accounts for (1) a reduced number of charged lipid beads per membrane area with increasing cholesterol concentration as well as a greater exposure of negative $\mathrm{PO}_{4}$ beads to the solvent. The latter may explain why cations and positive residues become more favorable attracted to bilayers with increasing cholesterol content.

\section{S3.2) Orientation of charged lipid beads as a function of cholesterol concentration}

The addition of cholesterol to bilayers may affect the structure of lipid head groups and, thus, how its partial charges are exposed to the solvent. The latter can, in turn, affect the electrostatic interaction of bilayers with amyloid fibrils. Accordingly, in the umbrella model, the backbone structure of lipid head groups changes in order to protect cholesterol from being exposed to the solvent. However, a more recent study in which all-atom simulations were compared with NMR experiments showed that the backbone structure of lipid head groups is not expected to change with increasing cholesterol concentration [5]. To study the effect of cholesterol on lipid head groups, we show in Fig. S3b, the distribution of the angle $\alpha$ between $\mathrm{NC}_{3}, \mathrm{PO}_{4}$, and $\mathrm{GL}_{1}$ beads - see inset. The distribution of this angle does not change significantly with increasing cholesterol concentration. This shows that the structure of DPPC head groups is not affected by the presence of cholesterol in the Martini force field. This is consistent with the behavior of the all-atom CHARM36 force field and NMR experiments. 

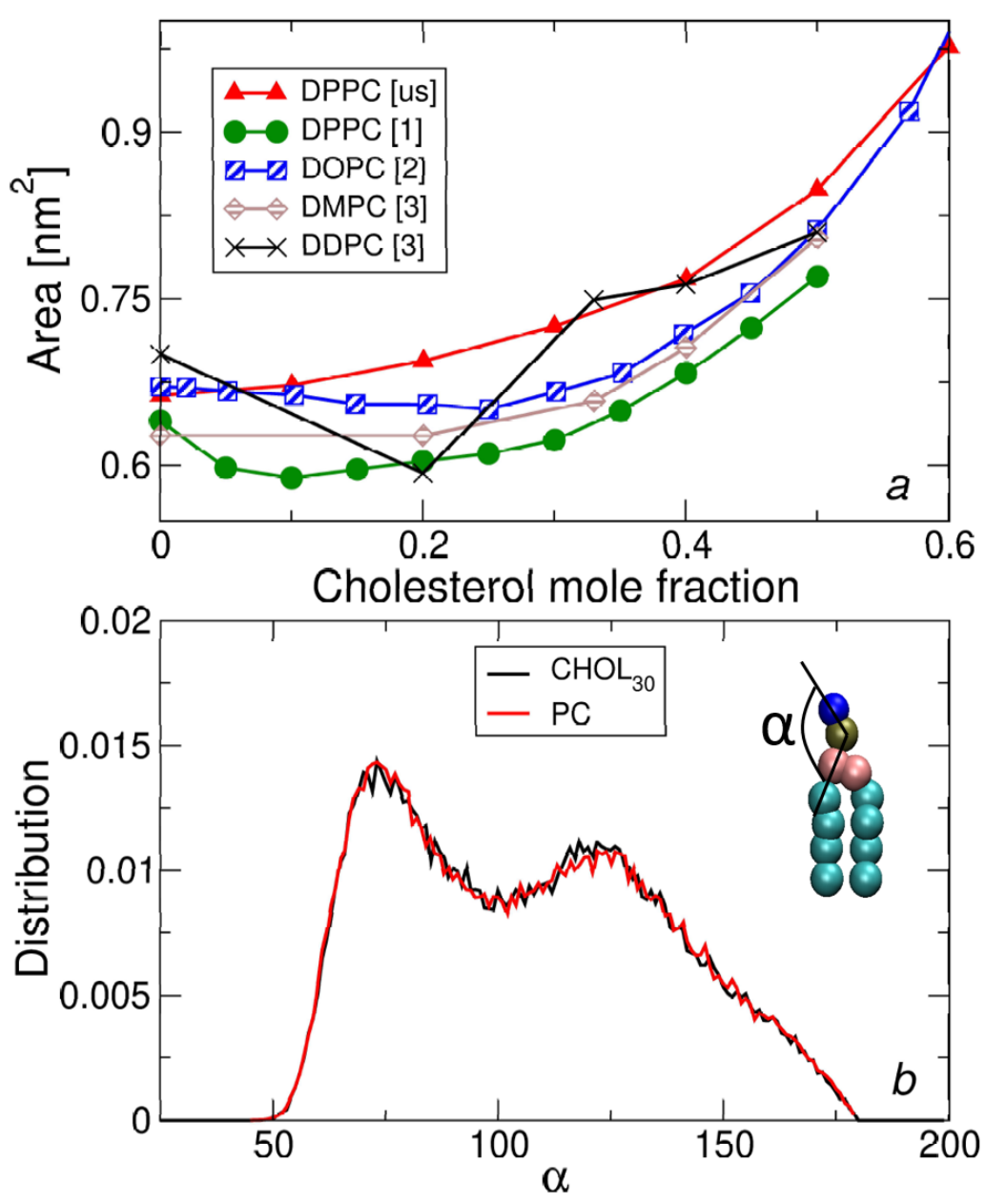

Fig. S4: Effects of cholesterol on the bilayer structure. (a) The average area of the lipid bilayer divided by the total numbers of $\mathrm{PC}$ lipids as a function of cholesterol mole fraction. Results from our Martini simulations are shown in red. (b) Distributions of the angle $\alpha$ between $\mathrm{NC}_{3}, \mathrm{PO}_{4}$, and $\mathrm{GL}_{1}$ beads for $\mathrm{PC}$ and $\mathrm{CHOL}_{30}$ bilayers.

[1] Olle Edholm and John F. Nagle, Biophysical Journal 89:1827-1832 (2005)

[2] Mohammad Alwarawrah, Jian Dai, and Juyang Huang, J. Phys. Chem. B 114:7519 (2010)

[3] Inna Ermilova and Alexander P. Lyubartsev, Soft Matter 15:78 (2019)

[4] M. Javanainen, A. Melcrová, A. Magarkar, P. Jurkiewicz, M. Hof, P. Jungwirth, and H. MartinezSeara. Chemical Communications, 53: 5380-5383 (2017).

[5] A. Catte, G. Mykhailo, M. Javanainen, C. Loison, J. Melcr, M.S. Miettinen, L. Monticelli et al. Physical Chemistry Chemical Physics 18:32560 (2016). 